\title{
Designing and Development of Media on Versatility of Neem
}

\author{
Kiranjot Sidhu \\ Department of Extension Education, Punjab Agricultural University, \\ Ludhiana 141 004, Punjab, India \\ E-mail: sidhs64@hotmail.com; neeluburj@yahoo.co.in
}

KEYWORDS Electronic; printed; validation; appropriateness

\begin{abstract}
One of the natural resources from the treasure house of plant kingdom is the Neem tree. This tree grows in various soil types and survives successfully under different weather conditions. The tree is virtually a gift of nature to mankind because of its multiple properties that have high utility value both at domestic and farm level beside its products being eco-friendly and bio-degradable. Gandhi and Patel (1997) conducted a survey in Andhra Pradesh, Punjab and Gujarat regarding the farmer's awareness about the impact of pesticides and reported it to be limited to the immediate surroundings. Use of alternative methods such as biological methods was practically non existent and awareness almost none. In this context media package on the topic "Versatile Neem for Domestic use" was designed and developed under the All India Coordinated Research Project on Home Science - Extension Component with the objective of translating the media into vernacular languages and using the same for disseminating information and knowledge on multiple qualities of Neem and its uses. The media package consisting of a booklet and a video film was designed and developed. The developed media both booklet and video film were then validated with the help of experts. The developed booklet and video film was found to be highly appropriate on selected characteristics. It was therefore recommended to be multiplied, translated and extensively used to promote the use of Neem .
\end{abstract}

\section{INTRODUCTION}

In India, plants have been traditionally used for agricultural practices and for homestead remedies. Their usage has been well documented in Vedas. They are as potent and effective today as they were during Vedic times. The influence of modernization, changes in life style and increased desire for quick remedies have however decreased the inclination for acceptance of time-tested medicinal properties of these plants. There has been further decrease in use of medicinal plants due to easy availability of chemically produced commercial products. These products are being presently used both at household and farm level though. The extent of use of pesticides/pest repellents by rural women at household level in Ludhiana districts was studied by Kaur (1996). She found that more than half of rural women were using common pesticides such as celphos, nepthalene balls, Baygon spray mats, BHC powder etc. There is a growing awareness that the use of chemical products can be hazardous in many ways. In view of this the steps need to be taken to reduce the use of hazardous chemicals and promote the use of available natural resources. Neem was reported by Hedge (1996) as well known plant for its medicinal properties in treating major disorder and diseases such as leprosy, lucoderma, jaundice, piles, chroniiculcer, diabetes, blood clots and hypertension. It also serves as home remedy for sore gums, tooth ache and endoparantes, ringworms, disinfectant and is used as a mouth wash. Apart from reducing dependence on allopathic medicine, Neem can also be used as spermicide and oral contraceptive to induce antifertility action in males.

Considering the above stated facts the need was felt to disseminate information and knowledge on the versatility of Neem. Enough data is available to support the effectiveness of well designed and developed media for transfer of information, knowledge and adoption of practices. Hence, the development of a media package was undertaken to develop and validate a media package on use of Neem for domestic purpose.

\section{METHODOLOGY}

The methodological framework adopted for developing the multi-media package has been explained under the appropriate headings and sub-headings.

Media package is a term that generally describes an instructional based package that integrates several forms of media that may include video, sound, animation, graphics and text. The following steps were followed:

Selection of Topic: Neem in Indian mythological sources is known as 'Sarva Roga Nivarini' 
meaning it can cure all diseases. This is further supported with the realities that Neem is used in Ayurveda, Unani and Homeopathy medicines due to its unlimited properties. All the parts of Neem tree have some medicinal properties which makes it capable to be named as 'Versatile Neem'.

The biodegradable properties of the Neem tree and the products prepared from different parts of the tree gives it an edge over chemically prepared products. The medicinal properties of Neem also take care of numerous health problems. In fact, all parts of the tree namely, the roots, trunk, bark, twigs, leaves, flowers, fruits, oil and gum have medicinal properties. The bitter taste of Neem is due to presence of numerous chemical compounds that supports the medicinal values present in all parts of the tree. Hence, there is a need to promote its use at domestic level..

Therefore, for creating general awareness and imparting knowledge among rural population regarding the versatility of Neem, the topic "Versatile Neem for Domestic Use" was selected for development of the media.

Selection of Content: Appropriate available literature sources such as books, magazines, newspapers, and journals etc, internet search sites were thoroughly explored for developing the content. The content thus selected was distributed among the experts to determine the correctness and completeness of the message. The improvements in the content were made as per the suggestions of the experts.

Organization of Content Material: To capture and sustain the interest of the users the content was transformed into manageable segments under the following headings:

1. Introduction

2. Qualities of Versatile Neem

3. Domestic products of Neem

4. Multiple Uses of Neem

5. Conclusion

This organized content was again distributed among the experts for judging the appropriate-ness of language which led to further editing of content.

Selection of Resource Media: The media were developed with the idea to accomplish a specific purpose through the best medium possible. Based upon the data on effectiveness of different media and to serve the basic purpose of highlighting the versatility of Neem, the following resource media were selected for inclusion in the package of media:
Printed Media (Booklet): Available literature was referred and discussion with experts were held in order to ascertain important attributes of a printed booklet. The format of booklet included text along with recognizable colorful photographs for accurate and easy understanding of contents. Puri (2001) in her study "Development of demonstration kit on nutrient enhancement of cereals and pulses" revealed that the developed demonstration kit consisting of booklet, photographs and actual specimens were effective for training rural women on nutrient enhancement of cereals and pulses.

Electronic Media (Video Cassette): The developed contents were transformed into video script. After the necessary modifications the video cassette was then developed on the basis of finalized script. The format used for developing script has been shown below:

\begin{tabular}{lccccc}
\hline Shot & Still & Shot & Duration & Audio & Music \\
No. & Video & description & of shot & & \\
\hline
\end{tabular}

A study by Neena (1997) on development of instructional message and corresponding educational slides for effective delivery of message on 'Food hygiene and sanitation' reported that there was significant gain in knowledge through synchronized slide-tape instructional sequence. Further, the visual images helped in enhancing knowledge significantly and thus they do have an edge over verbal messages.

Therefore, it was concluded that electronic media has significant value in motivating people for action and helping them in correct concept formation.

The video was shot at various locations and edited, dubbed and music was added to make the visuals more interesting.

Validation of Multi-Media Package: A panel of 10 judges, five each from the department of Entomology and Extension Education was selected for validation of the media developed. Their responses were rated regarding the developed package (booklet and video cassette) separately with respect to various characteristics to be considered for validation .

The scores were awarded to the various responses on three point scale. The responses were rated as appropriate, somewhat appropriate and not appropriate. These were assigned weightage of three, two and one score respectively. 
Analysis of Data: The scores were computed and means worked out. The items of indexes with mean score of 2 and above were considered valid. The mean score were calculated by using the following formula:

$$
\begin{aligned}
\overline{\mathrm{X}}=\frac{\Sigma \mathrm{Xi}}{\mathrm{N}} & \\
\text { Where } \overline{\mathrm{X}} & =\text { mean score } \\
\Sigma \mathrm{Xi} & =\text { sum of all observation score } \\
\mathrm{N} & =\text { Total no. of judges }
\end{aligned}
$$

Modification and Finalization: Each resource medium developed for the package was then revised/ modified on the basis of results of validation obtained through rating scale. The changes were made as per the suggestions of the experts on selected characteristics. The media were hence finalized.

\section{FINDINGS}

Appropriateness of Booklet: In order to examine the appropriateness of the booklet, 12 attributes were evaluated by the experts. The highest mean score of 3 was obtained by attributes such as title of the booklet and size of lettering while the lowest mean score (2.7) was secured by use of simple sentences and words. The mean of all the stated attributes was 2.7 and above. The overall mean score for the booklet was 2.85 which proved that booklet possesses all the qualities of an appropriate booklet. The results prove that the booklet was appropriate for the purpose for which it has been designed and developed. Pannu (2000) also reported that a standardized booklet can be effectively used for disseminating information on grain storage practices.

Table 1: Appropriateness of the developed booklet

\begin{tabular}{lc}
\hline Characteristics & Mean score (out of 3 ) $n=10$ \\
\hline Title & 3.0 \\
Size of lettering & 3.0 \\
Captions & 2.9 \\
Objective of learning & 2.9 \\
Colors used & 2.9 \\
Coverage of subject matter & 2.8 \\
Sequence of subject matter & 2.8 \\
Understandability of words & 2.8 \\
Pictures & 2.8 \\
Sentence length & 2.8 \\
Space between lines & 2.8 \\
Sentences and words & 2.7 \\
Overall score & 2.85 \\
\hline
\end{tabular}

Based upon the scores obtained after validation the sentences and words were simplified for better understanding. The other suggestions with regard to characteristics such as coverage and sequencing of subject matter were also incorporated for further improvement.

Appropriateness of Video Cassette: The appropriateness of video cassette was measured in terms of mean score as revealed in Table 2. The attributes of the video cassette i.e. content , understandability of language, music, sequence of information provided, clarity of message, cultural compatibility, video clarity, focus and sharpness of picture, duration and speed were judged by the judged and the mean scores were calculated.

Content scored highest with a mean value of 3.0 followed by music, sequence of information provided, clarity of message and cultural compatibility with a very high mean of 2.9. The speed of the video, scored the least (2.6 mean score). However, the mean overall mean of 2.84 proves that the video developed for the purpose of disseminating knowledge on domestic use of Neem was appropriate on basis of the selected characteristics. Garg (2001) tested the video cassette prepared for imparting knowledge on utilization of soybean and found it to be understandable and adequate for the purpose.

Table 2: Appropriateness of the developed video cassette

\begin{tabular}{lc}
\hline Characteristics & Mean score (out of 3) $n=10$ \\
\hline Content & 3.0 \\
Understandability of language & 2.9 \\
Music & 2.9 \\
Sequence of information provided & 2.9 \\
Clarity of message & 2.9 \\
Cultural compatibility & 2.9 \\
Video clarity & 2.8 \\
Focus and sharpness of picture & 2.8 \\
Duration & 2.7 \\
Speed & 2.6 \\
Overall score & 2.84
\end{tabular}

The improvements in the video were made especially by reducing the speed and increasing the duration.

\section{SUGGESTIONS}

The development of media is a time consuming task. However, effectiveness of both electronic and printed media in terms of creating awareness, generating interest and imparting knowledge has been proved time and again. But it is effective, only if designed and developed effectively and validated for the purpose for which it is prepared. Hence the developed and validated media should be extensively utilized by transla- 
ting into different regional languages to save on resources. Its intensive use by the field functionaries can help in effective and economical dissemination of information among the masses .

\section{REFERENCES}

Gandhi,V. P. and N.T. Patel.1997. "Pesticides and environment: A comparative study of farmers' awareness and behaviour in Andhra Pradesh, Punjab and Gujrat." Indian J. Agri. Econ., 52: 519-29.

Garg, B. 2001. Development of Instructional Material for Imparting Knowledge to Rural Women Regarding Utilization of Soyabean in their Diet. M.Sc. Thesis (Unpublished), Ludhiana: Punjab Agricultural University.
Hedge, N .1996. "Neem for all." Yojana, 40: 29-30.

Kaur, S. 1996. Extent of Use of Pesticides by Rural Women in Ludhiana District. M.Sc. Thesis (Unpublished), Ludhiana: Punjab Agricultural University.

Neena. 1997. Development of Instructional Message and Correspondence Educational Slides for Effective Delivery of Message on Food Hygiene and Sanitation. M.Sc. Thesis (Unpublished), Ludhiana: PAU.

Pannu, Kunwarjeet. 2000. Development of Educational Material on Grain Storage for Girl Members of Nehru Yuve Kendras' of Faridkot District. M.Sc. thesis (Unpublished), Ludhiana: Punjab Agricultural University

Puri, M. 2001. Development of Demonstration Kit on Nutrient Enhancement of Cereals and Pulses. M.Sc. Thesis (Unpublished), Ludhiana: Punjab Agricultural University. 